\title{
Bestimmung der Messunsicherheit mit der Monte Carlo Methode und Festlegung der Anzahl der benötigten Rechenläufe
}

\author{
Rüdiger Kessel \\ Physikalisch-Technische Bundesanstalt (PTB), Bundesallee 100, 38116 Braunschweig \\ Gerd Wübbeler \\ Physikalisch-Technische Bundesanstalt (PTB), Abbestraße 2-12, 10587 Berlin
}

Der ISO/DIN/BIPM Leitfaden zur Bestimmung der Unsicherheit beim Messen (GUM) gehört seit mehr als 15 Jahren zum Stand der Technik in der messtechnischen Praxis. Seit 2008 steht eine Erweiterung des GUM zur Verfügung, in der die Anwendung der Monte Carlo Methode auf die Messtechnik beschrieben wird. Nach einer kurzen Einführung in die Prinzipien der Monte Carlo Methode wird die Genauigkeit der Ergebnisse diskutiert, die von der Anzahl der durchgeführten Rechendurchläufe abhängt. Dabei werden die Schwächen eines adaptiven Verfahrens aufgezeigt, welches in der Erweiterung des GUMs für die Monte Carlo Methode beschrieben ist. Es wird ein alternatives Verfahren vorgestellt, welches mit einer fest vorgegebenen Anzahl für normalverteilte Ergebnisse eine vorgegebene Genauigkeit erreicht. Das dargestellte Verfahren führt zu einer verbesserten statistischen Sicherheit der Ergebnisse von Monte Carlo Simulationen. 